\title{
Speeding up the HMC algorithm: Some new results
}

\author{
Martin Hasenbusch* \\ Dip. Fisica dell'Università di Pisa and INFN, Largo Pontecorvo 2, I-56127 Pisa, Italy \\ E-mail: Martin.Hasenbuschedf.unipi.it
}

We give some new performance results for the Hybrid Monte Carlo (HMC) simulation of dynamical clover-improved Wilson fermions using an improved pseudo-fermion action. The generalisation of even-odd preconditioning for the standard Wilson fermion matrix to the clover-improved case is not unique. In the literature the so called symmetric and asymmetric versions are discussed. Most of the previous simulations of dynamical clover-improved Wilson fermions were done with the asymmetric version. Only recently, the JLQCD collaboration has pointed out that the symmetric version leads to a better performance of the HMC algorithm. Here, we show that also in combination with an improved pseudo-fermion action, the symmetric version of even-odd preconditioning leads to a better performance. For our lightest quark mass, which corresponds to $m_{P S} / m_{V} \approx 0.44$, we see a gain in performance by a factor of about 1.3 .

XXIIIrd International Symposium on Lattice Field Theory

25-30 July 2005

Trinity College, Dublin, Ireland

\footnotetext{
* Speaker.
} 


\section{Introduction}

Today, the standard method to simulated lattice QCD with dynamical fermions is the Hybrid Monte Carlo (HMC) algorithm [1] or related algorithms like the Polynomial Hybrid Monte Carlo (PHMC) or the Rational Hybrid Monte Carlo (RHMC) algorithm. Here we discuss improvements of the HMC simulation of clover-improved [2] Wilson fermions. In the following, for simplicity, we restrict ourself to the case of two degenerate flavours. In this case, configurations should be generated with a probability proportional to $B[U]=\exp \left(-S_{G}[U]\right) \operatorname{det} M[U]^{2}$, where $S_{G}[U]$ is the gauge action (in the following we shall use the Wilson gauge action) and $M[U]$ is the fermion matrix. The determinant is too expensive (cost $\propto$ Volume $^{3}$ ) to be evaluated in the numerical simulation. Therefore so called pseudo-fermions are introduced:

$$
\operatorname{det} M^{2}=\operatorname{det} M M^{\dagger} \propto \int \mathscr{D} \phi^{\dagger} \int \mathscr{D} \phi \exp \left(-\left|M^{-1} \phi\right|^{2}\right) .
$$

In order to facilitate a (non-physical) dynamics of the gauge-field, yet another auxiliary field is introduced: conjugate momenta $P$ for the gauge-field. The resulting Hamiltonian is

$$
\mathscr{H}(U, \phi, P)=S_{G}(U)+\left|M^{-1} \phi\right|^{2}+\frac{1}{2} \sum_{x, \mu} \operatorname{Tr} P_{x, \mu}^{2} .
$$

An update-step (often called trajectory) of the HMC algorithm is composed of

- Heatbath of the conjugate momenta $P$ and the Pseudo-fermion field $\phi$

- Evolution of $U$ and $P$ according to the equations of motion for some fixed time $t$ (in the following $t=1$ ), using a numerical integration method (e.g. the leapfrog scheme)

- Accept the resulting $U^{\prime}$ and $P^{\prime}$ with the probability $A=\min \left[1, \exp \left(-\mathscr{H}\left(U^{\prime}, \phi, P^{\prime}\right)+\exp (-\mathscr{H}(U, \phi, P)]\right.\right.$

Note that for an exact integration of the equations of motion, $\mathscr{H}\left(U^{\prime}, \phi, P^{\prime}\right)=\mathscr{H}(U, \phi, P)$, and hence the acceptance rate is 1 in this limit. Recent large scale simulations (see e.g. ref. [阿) with dynamical Wilson fermions were performed at rather large quark masses. It is debated whether, using chiral perturbation theory, the numerical results can be extrapolated to the physical quark masses. Therefore it would be highly desirable to reach lighter masses in the numerical simulation. However, the costs of the simulation increase rapidly as the quark mass decreases. The main reasons for this increase are the following: As the quarks become lighter the condition number of the fermion matrix increases. As a result, more iterations are needed to solve the linear systems of equations that need to be evaluated frequently in the HMC simulation. The second problem is that the step size of the integration scheme (e.g. leapfrog) has to be decreased with decreasing quark mass to maintain a constant acceptance rate.

Recently it has been demonstrated that the latter problem can be avoided or at least reduced by using alternatives [ [ , 6, 9, 7, 8, 9, 10] to the standard pseudo-fermions 1.1. Let us discuss in detail the approach of refs. [4, 5]: $N$ matrices $W_{i}$ are constructed such that $M=\prod_{i=1}^{N} W_{i}$. For each of these matrices a pseudo-fermion is introduced:

$$
\operatorname{det} M M^{\dagger} \propto \int \mathrm{D}\left[\phi_{1}^{\dagger}\right] \int \mathrm{D}\left[\phi_{1}\right] \ldots \int \mathrm{D}\left[\phi_{N}^{\dagger}\right] \int \mathrm{D}\left[\phi_{N}\right] \exp \left(-\sum_{i=1}^{N}\left|W_{i}^{-1} \phi_{i}\right|^{2}\right)
$$


The matrices $W_{i}$ should be chosen such that they have a smaller condition number than $M$. Our choice of the matrices $W_{i}$ is quite simple, we shift the fermion matrix by a constant, which corresponds to a larger fermion mass. For $N=2$, this results in $W_{1}=M+\rho$ and $W_{2}=(M+\rho)^{-1} M$. The obvious generalization to $N>2$ is given by

$$
W_{1}=M+\rho_{1}, \quad W_{i}=\left(M+\rho_{i-1}\right)^{-1}\left(M+\rho_{i}\right), \quad W_{N}=\left(M+\rho_{N-1}\right)^{-1} M .
$$

Preconditioning is a standard method in the solution of system of linear equations. The aim is to reduce the condition number of the matrix and this way to reduce the number of iterations needed to solve the problem. Even-odd preconditioning for the standard Wilson fermion matrix [11] relies on the fact that the hopping term $H$ connects only nearest neighbour sites on the lattice. As result, the fermion determinant can be expressed as $\operatorname{det} M=\operatorname{det} \hat{M}$ with $\hat{M}=1_{o o}-H_{o e} H_{e o}$, where $o$ indicates odd and $e$ even sites. Even-odd preconditioning can be easily combined with the alternative pseudo-fermion action discussed above, by replacing $M$ by $\hat{M}$ in eq. (1.4).

The generalization to the clover-improved case has been discussed in ref. [12]. Let us write the clover-improved Wilson matrix in the form $M=(1+T)-H$, where the clover-term is represented by the matrix $T$ which is diagonal in space-time. Using an even-odd decomposition of the lattice we can write the fermion matrix as

$$
M=\left(\begin{array}{cc}
1_{e e}+T_{e e} & H_{e o} \\
H_{o e} & 1_{o o}+T_{o o}
\end{array}\right)
$$

One can rewrite the fermion determinant either as (asymmetric even-odd preconditioning)

$$
\operatorname{det} M \propto \operatorname{det}\left(1_{e e}+T_{e e}\right) \operatorname{det} \hat{M}_{A S Y M} \text { with } \hat{M}_{A S Y M}=1_{o o}+T_{o o}-H_{o e}\left(1_{e e}+T_{e e}\right)^{-1} H_{e o}
$$

Or alternatively in a more symmetric form as (symmetric even-odd preconditioning)

$$
\begin{gathered}
\operatorname{det} M \propto \operatorname{det}\left(1_{o o}+T_{o o}\right) \operatorname{det}\left(1_{e e}+T_{e e}\right) \operatorname{det} \hat{M}_{S Y M} \\
\text { with } \hat{M}_{S Y M}=1_{o o}-\left(1_{o o}+T_{o o}\right)^{-1} H_{o e}\left(1_{e e}+T_{e e}\right)^{-1} H_{e o}
\end{gathered}
$$

In the HMC, for $\operatorname{det} \hat{M}_{S Y M}^{2}$ or $\operatorname{det} \hat{M}_{A S Y M}^{2}$ pseudo-fermions are used, while $\operatorname{det}\left(1_{o o}+T_{o o}\right)$ and $\operatorname{det}\left(1_{o o}+\right.$ $\left.T_{o o}\right)$ are exactly evaluated.

Here we should note that preconditioning not only reduces the effort required to solve the systems linear equations, but also has an effect on the step-size in the HMC simulation. E.g. in ref. [13] it had been noticed that for standard Wilson fermions, replacing $M$ by $\hat{M}$ in the pseudofermion action (1.1) allows to increase the step-size of the integration scheme by a factor of about 1.3 without decreasing the acceptance rate. Given this fact, one should check whether for cloverimproved Wilson fermions the use of $\hat{M}_{S Y M}$ or $\hat{M}_{A S Y M}$ in the pseudo-fermion action allows for a larger step size. Recently, the JLQCD Collaboration [3] has pointed out that, in combination with the standard pseudo-fermion action and the leap-frog integration scheme, the symmetric version (1.7) of the even-odd preconditioning is more efficient than the asymmetric one (1.6) that was used in most of the previous studies. In particular, the step-size, for fixed acceptance rate, is larger by a factor of 1.3 for the symmetric version than for the asymmetric one. In [5] we have only tested the improved pseudo-fermion action in combination with the asymmetric version (1.6) of the evenodd preconditioning. Here we study the combination of an improved pseudo-fermion action with the symmetric version (1.7) of even-odd preconditioning of the clover-improved Wilson fermion matrix. 


\begin{tabular}{|c|c|c|c|c|}
\hline scheme & $\rho$ & $N_{m d}$ & $P_{a c c}$, asymm & $P_{a c c}$, symm \\
\hline $\mathrm{L}$ & 0.5 & 25 & $0.770(3)$ & $0.847(5)$ \\
$\mathrm{S}$ & 0.5 & 10 & $0.883(3)$ & $0.934(2)$ \\
\hline
\end{tabular}

Table 1: Runs for the $8^{3} \times 24$ lattice at $\beta=5.2, c_{s w}=1.76$ and $\kappa=0.137$. $L$ indicates the leap-frog integration scheme, while $S$ indicates the partially improved scheme of Sexton and Weingarten. $\rho$ is the free parameter of the improved pseudo-fermion action, $N_{m d}$ the number of time-steps of each trajectory. $P_{a c c}$ is the acceptance rate.

\section{Numerical results}

In ref. [5] we found for extended runs on a $8^{3} \times 24$ lattice at $\beta=5.2, c_{s w}=1.76$ and $\kappa=$ 0.137 that autocorrelation times in units of trajectories do not depend, for fixed trajectory length $t$ and acceptance rate $P_{a c c}$, on the pseudo-fermion action and the integration scheme that is used. A similar result holds for the two dimensional Schwinger model [ $[$ ]. Also the simulation with 1500 trajectories of a $16^{3} \times 32$ lattice at $\beta=5.2, c_{s w}=2.0171$ and $\kappa=0.1358$ reported in [8] supports this observation. The simulations discussed in the following are sufficiently long to give reliable estimates for acceptance rates. However they are too short to provide sufficiently accurate estimates of autocorrelation times. Therefore, we have to rely, supported by the results reported above, on the assumption that the choice of the pseudo-fermion action has very little influence on autocorrelation times. In the following we have used the leap-frog integration scheme and, for most of the simulations, a partially improved scheme suggested by Sexton and Weingarten (see eq. (6.4) of ref. [16]). Comparing the performance of HMC simulations with these two schemes, one has to take into account that in one elementary step the variation of the action with respect to the gauge-field has to be computed once for the leap-frog scheme but twice for the partially improved scheme. Sexton and Weingarten [16] also proposed to use different step-sizes for different parts of the action. One should use a small step-size for the numerically cheap parts of the action and larger ones for the expensive parts. While in refs. [6, 7, 9, 10] different step-sizes are used for different parts of the pseudo-fermion action, we use a unique step-size for the whole pseudo-fermion action and a smaller step-size for the gauge action.

\subsection{Results for two pseudo-fermion fields}

As a first test, we simulated a $8^{3} \times 24$ lattice at $\beta=5.2, c_{s w}=1.76$ and $\kappa=0.137$. Results for the acceptance rates are given in table 1. The numbers for the asymmetric case (1.6) are taken from ref. [5]. For the symmetric case (1.7) we did not search again for the optimal value of the parameter $\rho$, but used the same value as in the simulations with the asymmetric preconditioning. We see that the acceptance rates are clearly larger for the symmetric preconditioning than for the asymmetric one.

\subsection{Results for 3 pseudo-fermion fields}

In ref. [8] it had been demonstrated that at least for small quark masses the performance of the HMC can be further improved by using 3 pseudo-fermion fields instead of 2 . Again the simulations were performed at $\beta=5.2$, however here we use $c_{s w}=2.0171$, which is the final result of the 


\begin{tabular}{|l|l|l|c|c|}
\hline$\kappa$ & $\rho_{1}$ & $\rho_{2}$ & $N_{m d}$ & $P_{a c c}$ \\
\hline 0.135 & 0.7 & 0.1 & 25 & $0.76(2)$ \\
\hline 0.1355 & 0.5 & 0.05 & 30 & $0.78(2)$ \\
\hline
\end{tabular}

Table 2: Runs for the $24^{3} \times 48$ lattice at $\beta=5.2$ and $c_{s w}=2.0171$.

\begin{tabular}{|c|l|l|c|c|}
\hline Precond. & $\rho_{1}$ & $\rho_{2}$ & $N_{m d}$ & $P_{a c c}$ \\
\hline Asymmetric & 0.4 & 0.03 & 25 & $0.809(6)$ \\
\hline Symmetric & 0.4 & 0.03 & 20 & $0.81(2)$ \\
\hline
\end{tabular}

Table 3: Runs for the $16^{3} \times 32$ lattice at $\beta=5.2, c_{s w}=2.0171$ and $\kappa=0.1358$.

ALPHA-collaboration [14] for the clover-coefficient. We performed simulations for $\kappa=0.135$, 0.1355 and 0.1358 . At these values of $\kappa$, the ratio of pseudoscalar and vector meson masses is $m_{P S} / m_{V} \approx 0.71,0.60$ [3] and 0.44 [15], respectively. Note that in the case of ref. [3] the simulations were not exactly performed at $c_{s w}=2.0171$ but at $c_{s w}=2.02$ instead. In ref. [3] for lattices of the size $20^{3} \times 48$, at $\kappa=0.134,0.135$ and 0.1355 using $N_{m d}=80,100$ and 160 an acceptance rate of $P_{a c c}=0.676(5), 0.666(6)$ and $0.678(7)$ had been obtained. $N_{m d}$ is the number of step of the leapfrog per trajectory. They have used the standard pseudo-fermion action with the symmetric even-odd preconditioning (1.7) and the leapfrog integration scheme. These numbers clearly show that with the standard pseudo-fermion action $N_{m d}$ has to be increased with decreasing quark mass to maintain a given acceptance rate. We have performed simulations on the slightly larger $24^{3} \times$ 48 lattice. We have used the partially improved integration scheme discussed above. We have generated about 200 trajectories after equilibrisation. The results are summarised in table 2 . It is not completely trivial to compare with ref. [3]; we use slightly larger lattices and our acceptance rate is larger. On the other hand there is some overhead in our simulations due to the additional pseudo-fermion fields. However, the main factor certainly due to the number $N_{m d}$ of steps needed for one trajectory. Taking these numbers we get $100 /(2 \times 25)=2$ and $160 /(2 \times 30)=2.6666$ in favour of the improved pseudo-fermions for $\kappa=0.135$ and 0.1355 , respectively. Note, that we have taken into account the fact that per step, the variation of the pseudo-fermion action with respect to the gauge-field has to be computed twice as often for the partially improved scheme as for the leapfrog scheme. Finally we compare with the simulation of a $16^{3} \times 32$ lattice at $\kappa=0.1358$ presented by the UKQCD collaboration in ref. [15]. The used the leapfrog scheme, asymmetric even-odd preconditioning and the standard pseudo-fermion action. They used $N_{m d}=400$. In ref. [8] we had already reported results for asymmetric even-odd preconditioning. Using the symmetric even-odd preconditioning we could further enlarge the step-size from $1 / 25$ to $1 / 20$. Taking into account the factor of 2 between the partially improved and the leapfrog scheme, we get a speed-up of a factor of 10 in favour of the improved pseudo-fermion action combined with the symmetric even-odd preconditioning of the clover-improved Wilson fermion matrix. 


\section{Conclusions}

We have studied the performance of the HMC algorithm simulating dynamical clover-improved Wilson fermions. In particular we have tested two different versions of the even-odd preconditioning of the clover-improved Wilson fermion matrix [12] in combination with an improved pseudofermion action [ [ , 5]. We find a clear advantage in favour of the symmetric version (1.7), as it is also the case for the standard pseudo-fermion action [3].

\section{References}

[1] S. Duane, A.D. Kennedy, B.J. Pendleton and D. Roweth, Hybrid Monte Carlo, Phys.Lett. B195 (1987) 216.

[2] B. Sheikholeslami and R. Wohlert, Improved Continuum Limit Lattice Action for QCD with Wilson Fermions, Nucl.Phys. B259 (1985) 572.

[3] JLQCD Collaboration, S. Aoki et al., Light hadron spectroscopy with two flavors of O (a)-improved dynamical quarks, Phys.Rev. D68 (2003) 054502, [hep-lat/0212039].

[4] M. Hasenbusch, Speeding up the Hybrid-Monte-Carlo algorithm for dynamical fermions, Phys.Lett. B519 (2001) 177, [hep-lat/0107019].

[5] M. Hasenbusch and K. Jansen, Speeding up Lattice QCD simulations with clover-improved Wilson Fermions, Nucl.Phys. B659 (2003) 299, [hep-lat/0211042].

[6] M. Peardon, Progress in lattice algorithms, Nucl.Phys.Proc.Suppl. 106 (2002) 3, [hep-lat/0201003]; M. Peardon and J. Sexton, Multiple molecular dynamics time-scales in Hybrid Monte Carlo fermion simulations, Nucl.Phys.Proc.Suppl. 119 (2003) 985, [hep-lat/0209037].

[7] QCDSF Collaboration, A. Ali Khan et al., Accelerating the Hybrid Monte Carlo Algorithm, Phys.Lett. B564 (2003) 235, [hep-lat/0303026].

[8] M. Hasenbusch, Full QCD Algorithms towards the Chiral Limit, Nucl.Phys.Proc.Suppl. 129 (2004) 27, [hep-lat/0310029].

[9] M. Lüscher, Schwarz-preconditioned HMC algorithm for two-flavour lattice QCD, [hep-lat/0409106].

[10] C. Urbach, K. Jansen, A. Shindler and U. Wenger, HMC algorithm with multiple time scale integration and mass preconditioning, [hep-lat/0506011].

[11] T.A. DeGrand and P. Rossi, Conditioning Techniques for Dynamical Fermions, Comput.Phys.Commun. 60 (1990) 211.

[12] K. Jansen and C. Liu, Implementation of Symanzik's Improvement Program for Simulations of Dynamical Wilson Fermions in Lattice QCD, Comput.Phys. Commun. 99 (1997) 221, [hep-lat/9603008].

[13] R. Frezzotti, M. Hasenbusch, J. Heitger, K. Jansen and U. Wolff, Comparative Benchmarks of full QCD Algorithms, Comput.Phys.Commun. 136 (2001) 1, [hep-lat/0009027].

[14] K. Jansen and R. Sommer, $O(\alpha)$ Improvement of Lattice QCD with two Flavors of Wilson Quarks, Nucl.Phys. B530 (1998) 185, [hep-lat/9803017].

[15] UKQCD Collaboration, C.R. Allton et al., Improved Wilson QCD Simulations with Light Quark Masses, Phys.Rev. D70 (2004) 014501, [hep-lat/0403007].

[16] J.C. Sexton and D.H. Weingarten, Hamiltonian Evolution for the Hybrid Monte Carlo Algorithm, Nucl.Phys. B380 (1992) 665. 